\title{
Characterization of a subsample of the Planck SZ source cluster catalogues using optical SDSS DR12 data
}

\author{
A. Streblyanska ${ }^{1,2}$, R. Barrena ${ }^{1,2}$, J. A. Rubiño-Martín ${ }^{1,2}$, R. F. J. van der Burg ${ }^{3}$, N. Aghanim ${ }^{4}$, \\ A. Aguado-Barahona ${ }^{1,2}$, A. Ferragamo ${ }^{1,2}$, and H. Lietzen ${ }^{5}$ \\ ${ }^{1}$ Instituto de Astrofísica de Canarias, C/Vía Láctea s/n, La Laguna, Tenerife, Spain \\ e-mail: alina@iac.es \\ 2 Universidad de La Laguna (ULL), Dept. Astrofísica, 38200 La Laguna, Tenerife, Spain \\ 3 IRFU, CEA, Université Paris-Saclay, 91191 Gif-sur-Yvette, France \\ ${ }^{4}$ Institut d'Astrophysique Spatiale, Université Paris-Sud, CNRS, UMR8617, 91405 Orsay, France \\ 5 Tartu Observatory, Faculty of Science and Technology, University of Tartu, Observatooriumi 1, 61602 Töravere, Estonia
}

Received 16 November 2017 / Accepted 1 June 2018

\begin{abstract}
Aims. The Planck catalogues of Sunyaev-Zeldovich (SZ) sources, PSZ1 and PSZ2, are the largest catalogues of galaxy clusters selected through their SZ signature in the full sky. In 2013, we started a long-term observational programme at Canary Island observatories with the aim of validating 500 unconfirmed SZ sources. In this work we present results of the initial pre-screening of possible cluster counterparts using photometric and spectroscopic data of the Sloan Digital Sky Survey DR12. Our main aim is to identify previously unconfirmed PSZ2 cluster candidates and to contribute in the determination of the actual purity and completeness of Planck SZ source sample.

Methods. Using the latest version of the PSZ2 catalogue, we selected all sources overlapping with the SDSS DR12 footprint and without redshift information. We validated these cluster fields following optical criteria (mainly distance with respect to the Planck pointing, magnitude of the brightest cluster galaxy, and cluster richness), and combined these criteria with the profiles of the Planck Compton $y$-maps. This combined procedure allows for a more robust identification of optical counterparts compared to simply crossmatching with existing SDSS cluster catalogues that have been constructed from earlier SDSS data releases.

Results. The sample contains new redshifts for 37 Planck galaxy clusters that were not included in the original release of PSZ2 Planck catalogue. We detect three cases as possible multiple counterparts. We show that a combination of all available information (optical images and profile of SZ signal) can provide correct associations between the observed Planck SZ source and the optically identified cluster. We also show that Planck SZ detection is very sensitive even to high- $z$ ( $z>0.5$ ) clusters. In addition, we also present updated spectroscopic information for 34 Planck PSZ1 sources (33 previously photometrically confirmed and 1 new identification).
\end{abstract}

Key words. large-scale structure of Universe - galaxies: clusters: general - galaxies: photometry - catalogs

\section{Introduction}

Massive galaxy clusters are an excellent tool to test cosmological models and to constrain cosmological parameters, for example dark matter or dark energy densities (e.g. Vikhlinin et al. 2009; Planck Collaboration XX 2014). In the last decade, the Sunyaev-Zeldovich (SZ) effect (Sunyaev \& Zeldovich 1972) has been used as a powerful technique to detect galaxy clusters. This effect produces a spectral distortion of the cosmic microwave background $(\mathrm{CMB})$ generated by the inverse Compton interaction between the CMB photons and the hot intracluster gas of electrons. Using this technique, the Planck ${ }^{1}$ satellite provided for the first time the possibility to detect galaxy clusters via the SZ effect in a full-sky survey (Planck Collaboration XXIX 2014; Planck Collaboration XXVII 2016).

\footnotetext{
1 Planck (http://www.esa.int/Planck) is a project of the European Space Agency (ESA) with instruments provided by two scientific consortia funded by ESA member states and led by Principal Investigators from France and Italy, telescope reflectors provided through a collaboration between ESA and a scientific consortium led and funded by Denmark, and additional contributions from NASA (USA).
}

The early SZ (ESZ) catalogue presented 189 clusters detected from the first 10 months of survey data (Planck Collaboration VIII 2011). In 2013, the Planck Collaboration released the first official version of the SZ catalogue, the PSZ1, which included 1227 sources detected during the first 15.5 months of observations (Planck Collaboration XXIX 2014). Finally, in 2015, the second catalogue PSZ2 (Planck Collaboration XXVII 2016) presented a complete list from the full-mission survey of 29 months. It contains 1653 sources ( 716 of them are unique for PSZ2). Even today, the PSZ2 catalogue is the largest SZ-selected sample of galaxy clusters with full-sky coverage.

The SZ surface brightness is independent of the redshift, but no information on the redshift of the source can be obtained from the signal. Yet, the abundance of clusters at high- $z$ are very sensitive to the cosmology (Borgani \& Guzzo 2001), so the identification of the counterparts of SZ clusters at other wavelengths are absolutely mandatory to make these catalogues scientifically useful. In this context, a few extensive followup programmes of the Planck Collaboration have been dedicated to confirm SZ sources using on-Earth and space facilities 
(Planck Collaboration Int. IV 2013; Planck Collaboration Int XXXVI 2016; Planck Collaboration Int. XXVI 2015).

Telescopes at Canary Islands Observatories are actively participating in the validation and characterization efforts of the ESZ and PSZ1 catalogues through the International Time Programme ${ }^{2}$ ITP12-2 and ITP13-08 (e.g. Planck Collaboration Int. XXXVI 2016; Barrena et al. 2018). Since 2015, we started the second twoyear long-term follow-up programme (LP15) at the Observatorio del Roque de los Muchachos to confirm new PSZ2 Planck cluster candidates and to measure their redshifts and other physical properties. The current paper is a first publication from the series of articles decicated to this follow-up campaign. In this paper, we present the results of the initial pre-screening of possible targets for this follow-up using the SDSS DR12 photometric and spectroscopic data available for PSZ2 targets. If a cluster counterpart is confirmed in the SDSS data, new imaging observations were not required in our LP15 programme and the cluster was directly considered for spectroscopy with the aim of obtaining its mean redshift, velocity dispersion, and dynamical mass. Whenever SDSS spectra were also available for several cluster members, we provided the spectroscopic redshift of the cluster based on those SDSS data and we considered it for a more detailed dynamical analysis. If the cluster was not confirmed through SDSS images, we included the cluster in our list of targets to retrieve deep imaging information in order to check the presence or absence of high- $z$ counterparts.

This paper is organized as follows. Sections 2 and 3 describe the Planck PSZ2 catalogue and SDSS data, respectively. Section 4 describes our methodology used for new cluster identification. In Sect. 5, we discuss the results of the PSZ2 characterization exposed in this work. Section 6 provides updated spectroscopic information for PSZ1 sources. Conclusions are presented in Sect. 7.

\section{The Planck PSZ2 cluster sample}

The SZ detection algorithms applied to make the PSZ2 catalogue areextended and refined versions of those used to construct the PSZ1 (Planck Collaboration XXIX 2014). As in the case of PSZ1 sources, the PSZ2 cluster candidates are blindly selected using three different detection methods: two multi-frequency methods based on the matched filter (MMF1 and MMF3) and the PowellSnakes $(\mathrm{PwS})$ procedure, which is based on a fast Bayesian approach to discrete object detection. The main PSZ2 catalogue contains all objects found by at least one of these three methods with a signal-to-noise ration $(S / N)>4.5$. The PSZ2 catalogue and the techniques used to construct it are described in detail in Planck Collaboration XXVII (2016). Some additional restrictions were imposed to avoid the spurious detections from the PwS algorithm and to remove detections that were confirmed to be spurious by the PSZ1 follow-up. In summary, the PSZ2 catalogue contains 1653 detections; 937 sources are common to PSZ1, while 716 are new; 291 of the PSZ1 catalogue are not in PSZ2). Of these objects, 1203 are confirmed clusters and 1094 have redshift estimates. Confirmation and redshift were obtained from PSZ1, cross-correlation with other catalogues (e.g. MCXC, ACT, SPT, RedMapper) and from the recent specific follow-up campaigns of different international teams (for detailed description see Sect. 7 in Planck Collaboration XXVII 2016). The main difference in the optical identification of the PSZ1 and PSZ2 sources in the SDSS region is the use of redMaPPer information (Rykoff et al. 2014; Rozo \& Rykoff 2014). In the v5.10 redMaPPer catalogue, 374

\footnotetext{
2 ITP: http://www $\cdot$ iac . es/eno $\cdot$ php?op1=5\&op2=13\&lang=en
}

sources were cross-identified with a cluster, while further search of counterpart in SDSS data with the redMaPPer algorithm confirmed 17 additional sources (see Sect. 3).

\section{Previous SZ follow-up using SDSS data}

The SDSS data covers most of the northern extragalactic sky and provides optical images and spectra between 3800-9200 A. Several cluster catalogues have been constructed using different data releases and detection algorithms (Hao et al. 2010; Wen et al. 2012). The optical validation of SZ sources in the PSZ1 catalogue has been carried out mainly through the cross-correlation with the cluster catalogue by Wen et al. (2012), which used the DR8 version of the SDSS data (Aihara et al. 2011). However, the redMaPPer cluster catalogue (Rykoff et al. 2014), using the DR8 release as well, contains many more clusters and has proven to be very efficient at optically identifying PSZ1, and later PSZ2 clusters.

The method based on the redMaPPer algorithm detects clusters by selecting spatial overdensities of galaxies belonging to the red sequence of the cluster candidate. This procedure is performed in a $10^{\prime}$ matching radius and imposing some constraints relative to the richness and angular separation between sources. The algorithm provides photometric redshift information and richness estimates for all detected clusters. The newly released SDSSbased redMaPPer catalogue (v5.10) produced 374 high-quality matches in which the counterparts are consistent with Planck mass and position information. More than half of these clusters are common for other catalogues, such as WHL SDSS sources (Wen et al. 2012) or RXC X-ray sources (Piffaretti et al. 2011). However, for 95 new PSZ2 Planck sources, the redMaPPer catalogue provided the most robust external confirmation (redshift and position of the optical cluster).

Although the redMaPPer algorithm has proven to be very efficient, as all automatic methods, it has its own limitations. For example, low richness systems, in particular nearby clusters $(0.05<z<0.2)$ are missed because of the high thresholds for richness estimates. Additionally, distant clusters $(z>0.5)$ could be missed owing to problems with photometric errors of the faint galaxies. In fact, the PSZ2 catalogue includes 17 confirmed clusters for which the automated redMaPPer search fails, but individual case-by-case inspections yield real counterparts.

For all these reasons, before starting the observational programme to validate unknown PSZ2 sources at Canary Islands observatories optically, an initial pre-screening of the target list in the SDSS database searching for new optical counterparts is absolutely necessary. We present in this work the results of this prescreening with the advantage that, nowadays, using the DR $12^{3}$ data release we manage a larger sky coverage and many more galaxy spectra than in previous releases. Therefore, the new information presented in this work includes two well-defined parts. First, an optical validation of unconfirmed PSZ2 sources, and second, an update of the redshift information (going from photometric estimates to spectroscopic measurements) for the PSZ1 clusters.

\section{Methodology for the validation and characterization of PSZ2 sources}

The cross-correlation between positions of unconfirmed PSZ2 sources and the SDSS survey yields 114 SZ cluster candidates

\footnotetext{
http: //wWw. sdss.org/dr12/
} 
covered in the DR12 footprint. To validate these clusters, we designed a four step criterium detailed in the following subsections.

\subsection{Visual inspection on an individual basis}

Using the SDSS DR12 Navigate tool $^{4}$ we retrieved a $10^{\prime} \times 10^{\prime}$ image centred at the nominal location of the Planck SZ source. We visually inspected the image looking for possible galaxy overdensities and the presence of a bright cluster galaxy (BCG). An image with a field of view of $10^{\prime} \times 10^{\prime}$ is large enough to cover the expected uncertainty in the Planck detection, which is full width half maximum (FWHM) of $2^{\prime}-6^{\prime}$ (see Fig. 3 in Planck Collaboration Int. XXXVI 2016). Clusters and rich groups at $0.1<z<0.4$ can be easily identified in the SDSS RGB images as a concentration of galaxies of the same colour. This first step, the visual inspection, is particularly important in the detection of clusters with $z>0.5$. The SDSS photometric survey is $95 \%$ complete at a magnitude limit of $r^{\prime}=22.2$. This means that only BCGs and a few additional galaxy members are visible in clusters at $z>0.5$. In addition, faint galaxies present larger photometric errors and thus redshift uncertainties. Therefore, distant clusters can be easily missed by automatic algorithms, but an inspection by eye can easily detect these systems.

Some SDSS images show problems with the presence of very bright stars, which make the automatic detection of sources almost impossible. In some cases, the SDSS database does not supply photometric information, mainly because the field is located in the edge of the surveys. Owing to these facts, we removed 10 sources from the initial list of 114 cluster candidates. These cluster fields are considered to be observed by our follow-up programme (LP15) with the Canary Islands telescopes.

\subsection{Inspection of Compton y-maps}

The full-sky Compton parameter maps ( $y$-map) obtained by the Planck Collaboration using the NILC and MILCA procedures (Planck Collaboration XXI 2014) are publicly available since 2015 (Planck Collaboration XXII 2016). These maps, constructed from linear combinations of the individual Planck frequency charts, preserve the SZ signal and cancel the influence of the CMB. These maps have been widely exploited in the literature. For example, they are used to obtain an accurate measurement of the SZ power spectrum at intermediate and large angular scales (Planck Collaboration XXII 2016) or to study the SZ effect using higher order statistical estimators such as the skewness and bispectrum. The $y$-map is also used to extract the SZ signal on regions centred at cluster positions and, in particular, to perform stacking analyses (Planck Collaboration XXII 2016). These $y$-maps also reveal the diffuse emission at the outskirts of clusters and in bridges between merging systems (Planck Collaboration Int. VIII 2013). Moreover, $y$-maps show SZ signatures even for poor galaxy clusters and they help in the confirmation of cluster candidates from PSZ1 catalogue (Planck Collaboration Int. XXXVI 2016; Barrena et al. 2018).

In Fig. 1 we present two typical examples of coincidences between the observed SZ profile and optical images for rich optical clusters. The $y$-map shows two clear peaks well above the noise level corresponding to two real optical clusters located at angular distance of $\sim 50^{\prime}$. One is the alreadyconfirmed cluster PSZ2 G066.41+27.03 (WHL J269.219+40.13, Planck Collaboration Int. XXVI 2015). After exploration of the

4 skyserver.sdss.org/dr12/en/tools/chart/naviinfo.aspx
SDSS images we were able to confirm the second source, PSZ2 G066.34+26.14.

Although $y$-maps are a valuable source of information for validation works, they must be used with care. It is known that the SZ reconstruction might be influenced by the Galactic dust radio emission. In some cases, dust emission may enhance the SZ signal, creating spurious detections. Such situations can be partially controlled by the measurements of Galactic extinction in the considered area, and by visual examination of the optical (mainly $g$ band) images and $y$-map shapes. In case of strong contamination by Galactic dust, the distribution of the signal in the $y$-maps is not compact and shows elongated profiles without a clear peak around the nominal position of the Planck SZ source. In many cases, the elongation of the $y$-map contours agrees well with the direction of Galactic dust structures visible in the $g$-band image. Barrena et al. (2018), Planck Collaboration Int. XXXVI (2016), and van der Burg et al. (2016) confirmed this fact by classifying a large amount of PSZ1 targets as "non-detections", most of which are located at low Galactic latitudes and showing high SZ significance, even $S / N>7$ in some cases (for example PSZ1 G071.64-42.76). Figure 2 presents three examples of $y$-maps for the PSZ2 targets where no optical counterparts have been identified. The possibility of counterparts associated with high redshift clusters is not excluded, but only deeper optical images would confirm or reject this fact. However, the irregular contour profiles observed in $y$-maps and its morphological coincidence with the structure observed at $857 \mathrm{GHz}$ (thermal dust emission) suggest that the most probable scenario is a false SZ detection.

Taking into account all facts outlined above, we inspected the $y$-map (obtained through the MILCA algorithm) of each PSZ2 source paying special attention to the profile shape, SZ signal, and intensity above the noise level $\left(>3 \times 10^{-6}\right.$ in units of the corresponding $y$-map).

\subsection{Cluster red sequence}

We retrieved SDSS DR12 photometry for each field and the photometric redshift information of galaxies using SQL Search $^{5}$. We inspected colour-magnitude diagrams (see Fig. 3) $\left(g^{\prime}-r^{\prime}, r^{\prime}\right)$ and $\left(r^{\prime}-i^{\prime}, i^{\prime}\right)$ looked for the cluster red sequence (CRS; e.g. Gladders \& Yee 2000). We fit the red sequence considering all galaxies with colours in the range \pm 0.05 respect the colour of the BCG. This allows us to derive the photometric redshift of the galaxy overdensities following prescriptions detailed in Planck Collaboration Int. XXXVI (2016), Sect. 4.2. After identifying the galaxy clumps and deriving their $z_{\text {phot }}$, we evaluated the richness of these systems to validate the galaxy overdensities as actual SZ counterparts. We detail this process in the following subsection.

\subsection{Richness}

The SZ clusters of the Planck catalogue are massive structures (see e.g. Figs. 28, 29 in Planck Collaboration XXIX 2014). Assuming that cluster richness can be understood as optical mass tracer (e.g. Rozo et al. 2009b), galaxy clusters associated with Planck SZ emission should also present rich galaxy cluster populations. Therefore, we can estimate the richness of the galaxy population in our optical cluster sample to validate our SZ detection.

Rozo et al. (2009a, b) proposed a robust richness estimator from optical catalogues assuming a matched filter model for the

skyserver.sdss.org/dr12/en/tools/search/sql. aspx 


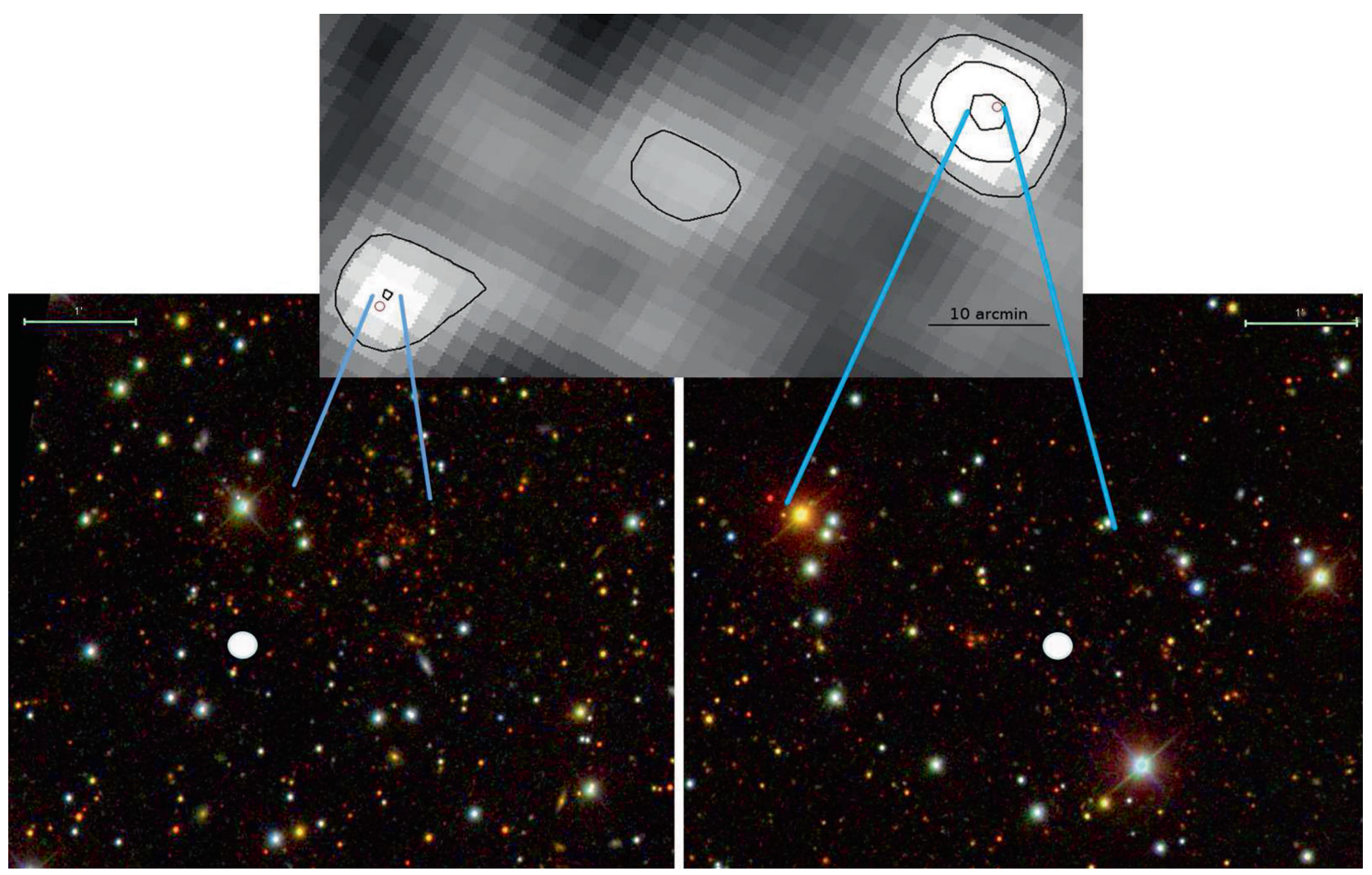

Fig. 1. MILCA Compton $y$-map of the area around two confirmed SZ sources, PSZ2 G066.34+26.14 (left, confirmed in this paper, SZ S/N=5.6) and PSZ2 G066.41+27.03 (right, validated in Planck Collaboration Int. XXVI (2015), SZ S/N=8.8), superimposed to a zoomed RGB SDSS images. The black contours correspond to the $3,6,9 \times 10^{-6}$ levels of the Compton $y$-map in this area. The single black contour in the middle of the $y$-map corresponds to a noise fluctuation. The red circles indicate the nominal PSZ2 source locations. In the RGB SDSS images this nominal position is indicated with white filled circles. Both PSZ2 sources show the presence of clusters at similar high $z, z_{\text {phot }}=0.62$ and 0.53 , respectively. Blue lines delimit the area corresponding to the peak of SZ signal for PSZ2 G066.34+26.14, which enclosed almost all cluster members. Scale bars in RGB images are $1^{\prime}$.
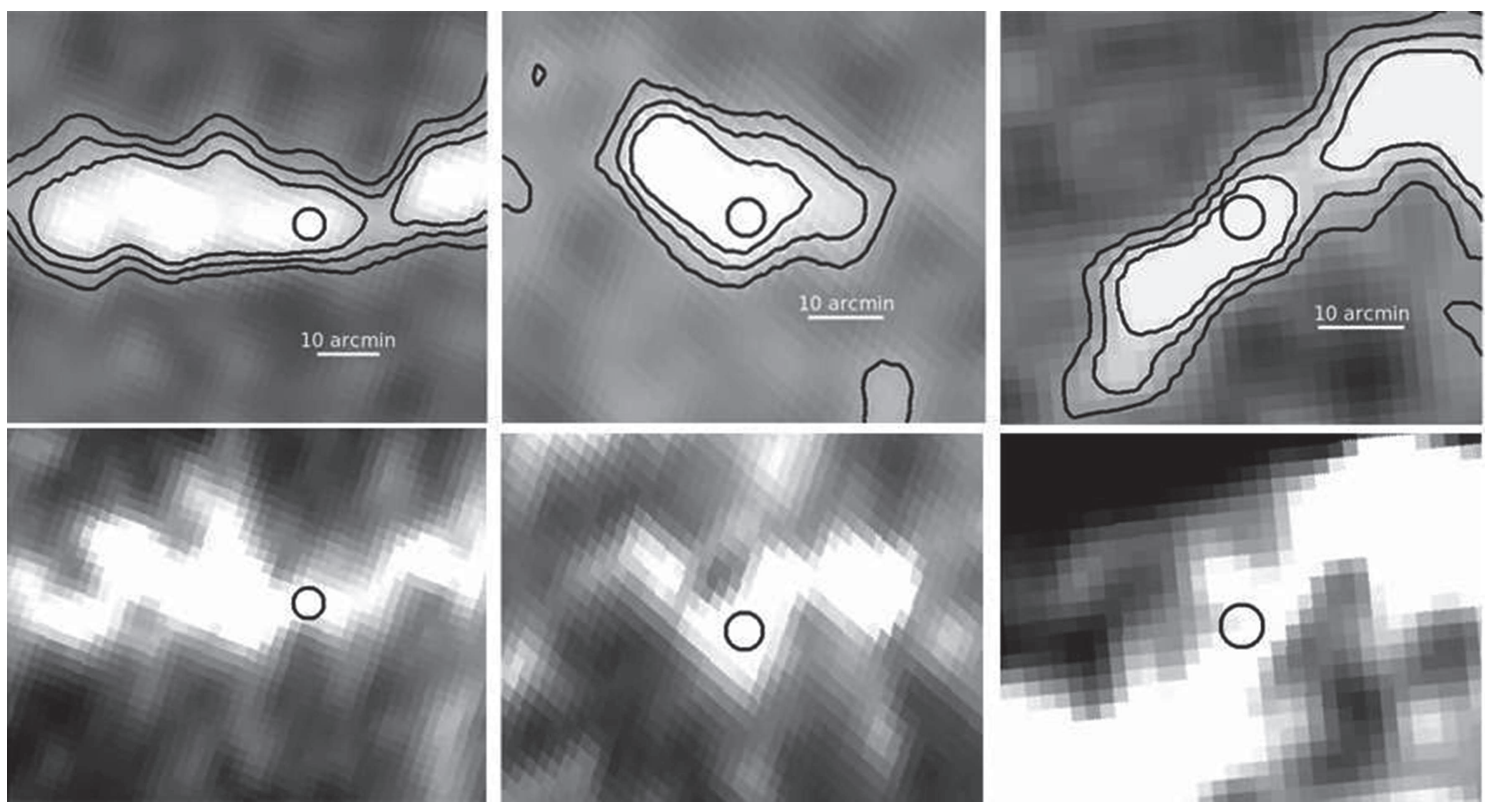

Fig. 2. Three examples of Planck clusters in which the SZ signal seems to be strongly influenced by Galactic dust contamination. From left to right three columns correspond to PSZ2 G029.87-17.81, PSZ2 G148.60-48.61, and PSZ2 G179.33-22.22, respectively. We present for each source Compton $y$-map (top row) together with the corresponding Planck $857 \mathrm{GHz}$ map (bottom row). These clusters have $S / N \sim 5$ in the PSZ2 catalogue. Black contours correspond to the 3,6 and $12 \times 10^{-6}$ levels of the Compton $y$-maps in this area. No obvious peak at the nominal position of Planck sources (showed as black circles) is evident in these three cases. The $857 \mathrm{GHz}$ map for each source suggests that most of the emission arises from dust. The SZ flux profile follows an elongated structure that coincided with the direction of a dust Galactic filament. While the possibility exists that cluster counterparts correspond to high-z clusters, in the absence of deeper data we classify these as false SZ detections. 


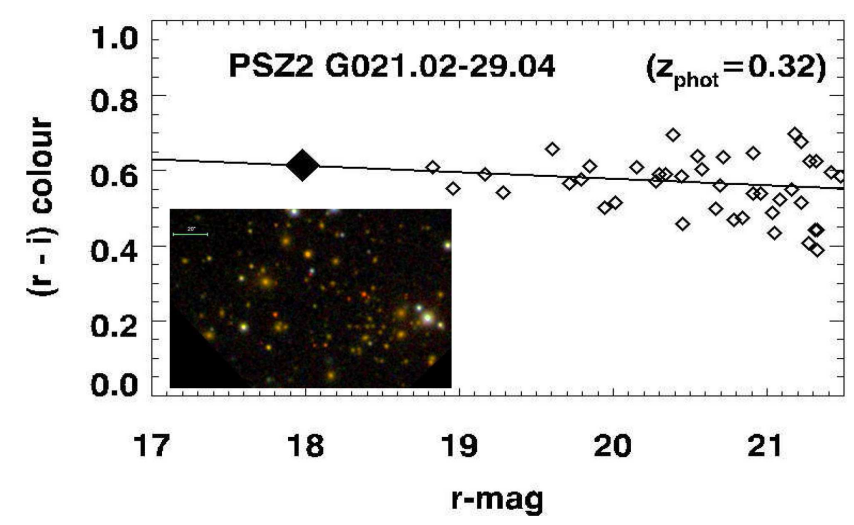

Fig. 3. Example of the colour-magnitude diagram using data for cluster PSZ2 G021.02-29.04. The red sequence (solid line) is consistent with a photometric redshift of 0.32 according to the relation of Planck Collaboration Int. XXXVI (2016). We consider galaxies with a colour within \pm 0.05 from the red sequence as likely cluster members.

density profile of galaxies. This method is part of the redMaPPer algorithm and proposed to be applied in photometric catalogues that sample the galaxy population of the systems. However, our sample includes clusters at intermediate redshift, where only the brightest galaxies are visible, making it very difficult to find confidence models to the galaxy density profiles. In addition, we consider SDSS photometry, which is not a very deep photometric survey.

A simple prescription to estimate the richness of a cluster is to compute the actual number of cluster galaxies in a magnitude range within the virialized region. Using this classical method, we basically count galaxies in a magnitude range within an aperture, adding the colour information to minimize non-cluster members. This method was introduced by Abell (1958). More recently, Wen et al. (2012) also followed a similar technique to construct their cluster catalogue.

For a given cluster with a photometric redshift $z_{\text {phot }}$, we counted likely cluster members (assumed as galaxies within $\pm 0.05)$ of the $z_{\text {phot }}$ of the cluster showing $r$-magnitudes in the range $\left(r_{\mathrm{BCG}}, r_{\mathrm{BCG}}+2.5\right)$ within the $0.5 \mathrm{Mpc}$ from the cluster centre. For this task we considered as a BCG the most luminous galaxy inside the identified likely cluster members. In order to obtain the background subtracted richness and decontaminate from the galaxy field contribution, we computed galaxy counts in a $10 \times 10$ degrees region (centred at $\mathrm{RA}=10: 28: 00, \mathrm{DEC}=55: 20: 00$ ), where SDSS DR12 photometric sample presents a homogeneous total coverage. We applied the same procedure as for the cluster region (assuming the same $z_{\text {phot }}$ and $r$-magnitudes ranges), thereby creating a field galaxy sample or local background for each cluster. This field sample was scaled to the $0.5 \mathrm{Mpc}$ area and subtracted from the cluster counts to obtain a statistical estimate of the number of galaxies for each cluster.

However, one of the main problems in estimating the richness in clusters at medium-high redshift $(z>0.3)$ is the magnitude limit of the photometric sample. Given that SDSS DR 12 sample is $95 \%$ complete ${ }^{6}$ down to $r^{\prime}=22.2$, we restricted this study to $z<0.6$. Beyond this redshift the $r_{\mathrm{BCG}}+2.5$ magnitude is higher than the magnitude limit $r=22.2$. Another source of underestimation of richness is the faintness of the galaxy clusters at medium-high redshift. Possible errors in $z_{\text {phot }}$ estimates of faint galaxies may introduce large uncertainties in the cluster counts, and therefore

\footnotetext{
6 http://wwW. sdss.org/dr12/scope/
}

make a robust richness estimate very difficult. Nonetheless, we considered the richness an indicative parameter to distinguish between the real and false counterparts at $z<0.6$.

The mass-redshift distribution of the Planck SZ sources is described in Planck Collaboration XXXII (2015), which provides the detectable mass of SZ sources in the PSZ1 catalogue. Following this relation, no poor systems could be considered actual counterparts even if they are well aligned with the Planck pointing. However, the Planck CMB and $y$-maps contain noise, making it possible that a significant percentage of low-mass haloes scatter over the SZ detection threshold. This effect is known as Eddington bias (Eddington 1913). van der Burg et al. (2016) analysed this effect and confirmed $19 \mathrm{SZ}$ clusters at $z>0.5$ with $\mathrm{M}_{500} \sim 2 \times 10^{14} M_{\odot} h_{70}^{-1}$. Moreover, nearby $(z<0.2)$ galaxy systems may also be detected in the Planck SZ maps even if they have masses $\mathrm{M}_{500} \sim 10^{14} M_{\odot} h_{70}^{-1}$. Clusters with lower masses are not expected to be actual SZ counterparts.

We studied the richness (as mass tracer) of two poor galaxy clusters to find an appropriate threshold to separate real from false counterparts. These are Abell 725 and Abell 796, at $z=0.092$ and 0.157 , which present global velocity dispersions of $\sigma_{v}=534 \pm$ $115 \mathrm{~km} \mathrm{~s}^{-1}$ and $586 \pm 160 \mathrm{~km} \mathrm{~s}^{-1}$, respectively (Boschin et al. 2008). The mass estimates of these systems show $\mathbf{M}_{500} \sim 3 \times$ $10^{14} M_{\odot} h_{70}^{-1}$, so they are low-mass clusters. By calculating the richness, as defined above, we find that Abell 725 presents 8 cluster members within a region of $0.5 \mathrm{Mpc}$ radius in the magnitude range $\left(r_{\mathrm{BCG}}, r_{\mathrm{BCG}}+2.5\right)$. In the same way, Abell 796 presents a richness of 5 galaxies. Therefore, taking into account these numbers, we can conclude that clusters can be considered actual SZ counterparts if they present a richness at least of 5 galaxies, according to our richness definition.

In order to evaluate the chance associations of galaxies to SZ targets, we measured the richness at 10000 positions randomly distributed in the $10 \times 10$ degree background field and imposed the restrictions on magnitude, colours, and apertures according to different redshifts. We find about $15 \%$ probability of finding overdensities with $\mathrm{R}=1$, while the probability of finding overdensities with $\mathrm{R}=5$ by chance is below $0.1 \%$. Moreover, the combination of the richness threshold with the other criteria, as distance to the Planck pointing, ensures that the association to Planck source is robust.

In order to make our sample selection uniform and taking into account the large photometric errors for faint distant sources, we assumed this richness cut for high-z clusters as well. However, we noticed that most of our high-z clusters $(z>0.4)$ have $\mathrm{R}$ higher than 5, following the expected trend.

This threshold on $\mathrm{R}$ imposes a necessary, but not sufficient condition to validate the SZ counterparts. Although some SZ sources could potentially have values $\mathrm{R}<5$, our conservative criteria ensures that those candidates validated with the $R>5$ condition are bona fide optical SZ counterparts.

\subsection{Spectroscopic data}

In addition to the photometric information, we also used the SDSS DR12 spectroscopic information available around the Planck PSZ2 source positions. We also used the SDSS-III photometric cluster catalogue by Wen et al. (2012), the group galaxy catalogue constructed by Tempel et al. (2014) of low redshift candidates, and the DR12 BOSS-CMASS galaxy list for the high redshift clusters. We complemented this information with some spectroscopic results obtained with the validation programmes (Planck Collaboration Int. XXXVI 2016). Given that PSZ2 counterparts show a few galaxy members with spectroscopic redshifts, 



Fig. 4. Left: apparent BCG $r$-band magnitude versus redshift of the complete sample of 54 sources studied in this work. The dashed curve corresponds to the simple prescription $M_{r}^{*}-1.5$. Right: cluster optical centre offsets relative to their Planck SZ position as a function of cluster redshift for a sample of 51 sources. Cases with multiple optical counterparts have been excluded from this analysis. The dashed horizontal line is $5^{\prime}$, which represents the maximum offset expected for a Planck SZ detection (i.e. $2 \times$ FWHM Planck multi-frequency combined beam). The dotted line corresponds to the physical $1 \mathrm{Mpc}$ radius region at the corresponding redshift. The black dots corresponds to a sample of 34 clusters from Table A.1. These sources fulfil our distance and richness constraints (see Sect. 4). The open squares correspond to 17 clusters that were classified as "potential optical counterparts" (Table A.2).

the spectroscopic information was only used to determine the exact redshift of the cluster.

\section{Results for the PSZ2 catalogue}

Our validation and characterization procedure is based on the simultaneous use of optical images and Compton $y$-maps. By analysing already-confirmed PSZ1 and PSZ2 sources, we find that, in most cases, there is a strong dependence between the location of the optical counterpart and the intensity, shape, and size of the SZ signal in the $y$-maps especially for high $\mathrm{S} / \mathrm{N}$ clusters. This dependence could be extrapolated to still unconfirmed sources, and could help to confirm less rich and less concentrated clusters.

Using this combined approach, we were able to identify optical counterparts for 54 new Planck PSZ2 cluster candidates.

In Fig. 4 (left plot) we present the apparent $r$-band magnitudes of the bright central galaxies (BCGs) versus redshift of the complete sample of 54 sources studied in this work. Following Menanteau \& Hugues (2009), the dashed curve corresponds to the simple prescription $M_{r}^{*}-1.5$, and assuming passive evolution with redshift. The values for $M_{r}^{*}$ are taken from Blanton et al. (2003) and the effective wavelengths for each SDSS filter are taken from Table A.1 in Blanton \& Roweis (2007). As expected, all the identified BCGs identified show magnitudes brighter than $M_{r}^{*}-1.5$, which represents a necessary condition for a correct cluster identification.

In addition, we take into account the distance between optical centre and the nominal Planck SZ coordinates to obtain a robust confirmation of optical counterpart. The position error predicted for SZ detections in the Planck SZ maps is about $2^{\prime}$ for targets in the PSZ1 sample (Planck Collaboration XXIX 2014; Barrena et al. 2018). Therefore, optical counterparts are not expected to be found beyond 2.5 times the beam FWHM, which means that the cluster associated with the SZ effect should be closer than $\sim 5^{\prime}$ from the SZ PSZ1 source coordinates. However, when the clusters are nearby systems $(z<0.2)$ their apparent radius may fill a large region, and in these cases their centre offsets relative to their SZ position may be larger. Figure 4 (right panel) shows the distance of the 51 cluster optical centres to the nominal PSZ2 coordinates. No optical counterparts, except PSZ2 G231.41+77.48 and PSZ2 G084.69-58.60, are found beyond 5'

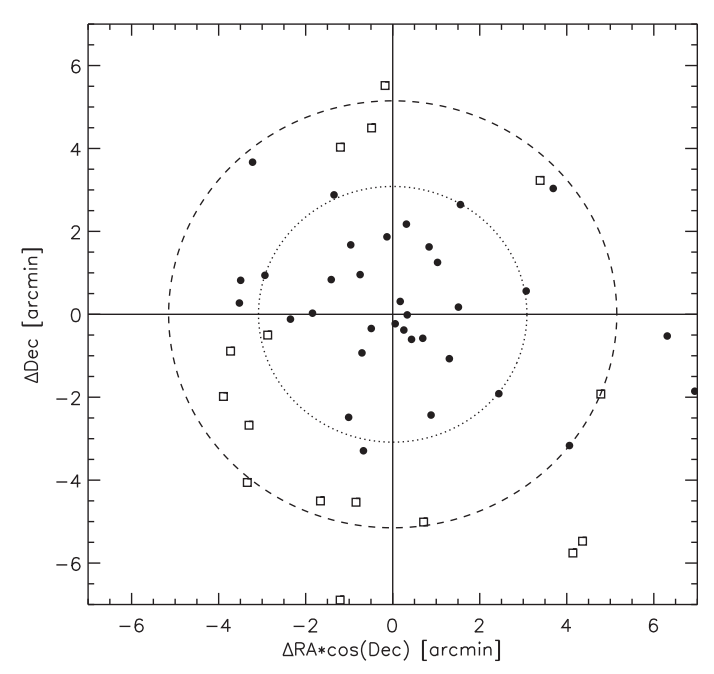

Fig. 5. Distribution of the optical centre offsets relative to their Planck SZ positions for the sample of 51 clusters. Thirty-four confirmed clusters included in Table A.1 (dots) and 17 clusters potentially associated with the corresponding PSZ2 source listed in Table A.2 (squares). Inner dashed line corresponds to 3.1 radius region, which encloses the $68 \%$ of the PSZ2 confirmed clusters. External dashed line encloses the $2 \times F W H M$ beam of the Planck SZ detection. Cases with multiple optical counterparts have been excluded from this analysis.

from Planck nominal pointing at $z>0.2$. We include these clusters as true counterparts because of their specific shape seen in $y$-map (see Fig. 8 and discussion below). Figure 5 shows the final offset distribution of cluster optical centre relative to their Planck SZ position. We find that $68 \%$ of the 37 confirmed cluster sample are enclosed within $3^{\prime} .1$, which is consistent with what is expected for SZ Planck target selection (Planck Collaboration XXIX 2014; Barrena et al. 2018).

In Table A.1 we summarize our results for the first subgroup of 37 sources (distance $<5^{\prime}$ or/and inside $1 \mathrm{Mpc}$ and richness $>5$ ), providing the official ID number, taken from the PSZ2 catalogue, the Planck Name, optical counterpart coordinates (assumed to be the BCG position or, in the absence of $\mathrm{BCG}$, as approximate geometrical centre of the likely members), redshift (photometric and, if available, spectroscopic), and 

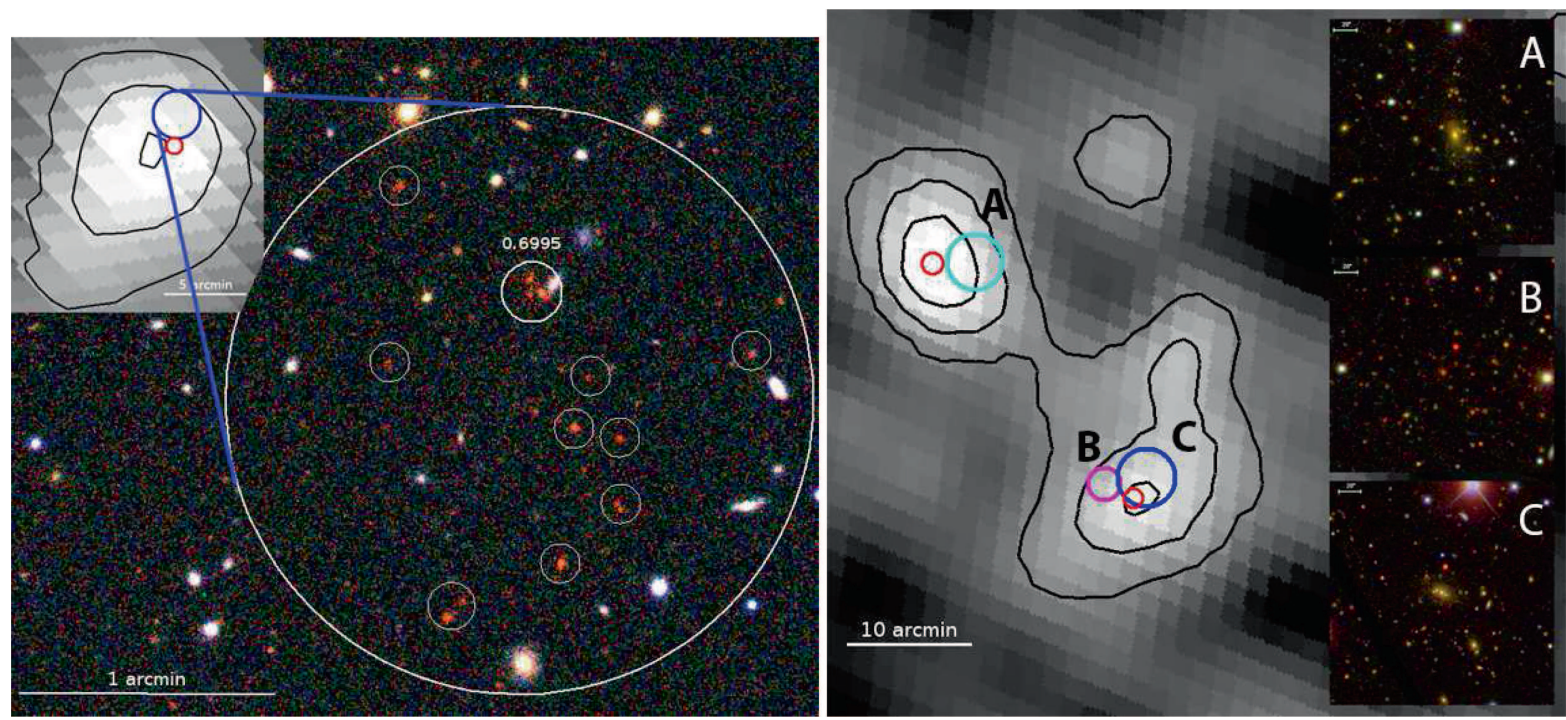

Fig. 6. Left: compton $y$-map and RGB SDSS image of PSZ2 G086.28+74.76. The black contours correspond to the 3,5 , and $6 \times 10^{-6}$ levels of the Compton $y$-map in this area. The red circle indicates the nominal PSZ2 position. Big white circle overlapping the RGB image corresponds to $1 \mathrm{Mpc}$ at $z=0.7$. We found that the SZ signal comes from a cluster at $z_{\text {spec }}$ of 0.699 . We find 12 likely galaxy members at $z_{\text {phot }} \sim 0.75_{0.08}^{+0.07}$ (small circles). The BCG is at $z_{\text {spec }}=0.6995$. Right: PSZ2 G270.78+36.83 (bottom) and PSZ2 G270.88+37.23 (top) are two sources separated by the distance of about $25^{\prime}$. We identify one counterpart for PSZ2 G270.88+37.23 at $z_{\text {phot }} \sim 0.23$ (cyan circle) and two counterparts for PSZ2 G270.78+36.83 at $z_{\text {phot }} \sim 0.52$ and $z_{\text {phot }} \sim 0.22$ (magenta and blue circles), respectively. The red small circles indicate the nominal PSZ2 source coordinates and the size of the circles corresponds to $1 \mathrm{Mpc}$ at the cluster redshift. We insert the zoomed RGB images of the centres of these clusters. Scale bar in the RGB images is $20^{\prime \prime}$.

number of spectroscopically confirmed galaxy members. We also provide spectroscopic information for 10 sources: 8 have spectroscopic coverage from the SDSS DR12 survey and, for 2 cases we quote the spectroscopic redshift from our longslit observations using ACAM spectrograph installed at the William Herschel Telescope (for a full description of the instrument set-up, see Planck Collaboration Int. XXXVI 2016). Multiple optical counterparts are identified for three SZ sources. We also provide richness information for each cluster. Most of our optical counterparts are clusters included in the catalogue of Wen et al. (2012). However, we update the position (attending to the BCG identifications) and redshift information. All clusters have $\mathrm{S} / \mathrm{N}$ of the $\mathrm{SZ}$ signal (using the MMF1, MMF3, or PwS detection pipelines) between 4.5 and 5.8 except PSZ2 G237.68+57.83 ( $S / N=7.4)$ and PSZ2 G153.56+36.82 $(S / N=15.9)$.

The second subgroup of 17 clusters does not fit the conditions of minimal distance and richness imposed to be considered actual optical counterparts linked to the SZ emission. This means that this group of clusters are beyond $5^{\prime}$ from the nominal Planck SZ centre or are not rich enough systems. However, because some of the Planck clusters from the PSZ1 catalogue were already detected at larger distances by the previous teams (see Planck Collaboration Int. XXXVI 2016; Barrena et al. 2018) and that these objects correspond to actual clusters in SDSS DR12 images, we decided to keep these sources for further detailed investigation. So, we refer to these sources as possible counterparts and present them in Table A.2.

In the rest of the cases, the cluster identification performed on SDSS DR12 imaging data was not conclusive. Those SZ targets may be associated with areas with strong dust contamination (thus producing false SZ detections in the Planck maps), or the corresponding cluster counterparts are high redshift clusters, and therefore not visible in the SDSS images because of their low depth. We will re-observe these fields during our follow-up in order to obtain deep images and confirm either the distant cluster or false SZ detection.

In the following, we describe, as examples, results for a few clusters at various redshifts.

PSZ2 G069.35-15.58 This is a low redshift system at $z_{\text {phot }} \sim 0.09$ (see Fig. 7). We obtain $\mathrm{R}=25$ for this cluster, which is one of the richness of the clusters studied in this work. We have acquired spectroscopic redshifts for two cluster members using ACAM/WHT long-slit spectroscopy under an International Time Programme (ITP13) of the ORM, obtaining a mean $z_{\text {spec }}$ of 0.095 .

PSZ2 G086.28+74.76 The importance of visual inspection of RGB images is clearly shown with this example (see Fig. 6, left panel). This is a distant cluster at $z_{\text {spec }}=0.6995$. As a result of this high redshift, the cluster galaxy population is very faint. The BCG has an apparent magnitude of $r^{\prime}=21.63$, so the SDSS catalogues only include photometry for a few cluster members, thus the red sequence is completely missing in the corresponding colour-magnitude diagrams. For this reason, the richness cannot be estimated. However, by eye inspection we are able to identify 12 likely cluster members with colours in agreement with a $z_{\text {phot }} \sim 0.75_{-0.08}^{+0.07}$. Moreover, the brightest galaxy (probably the BCG of this cluster) has a $z_{\text {spec }}=0.6995$ in the SDSS DR 12 spectroscopic catalogue.

PSZ2 G231.41+77.48 Even though this cluster is slightly beyond the limit of our accepted $5^{\prime}$ radius search $\left(5^{\prime} .7\right)$, the MILCA contours confirm that this cluster is our optical counterpart to the SZ signal (Fig. 8). We found two spectra (including the $\mathrm{BCG})$ at $z_{\mathrm{spec}} \sim 0.346$. A similar situation appears with PSZ2 G084.69-58.6, which shows a similar shift between the peak of the SZ signal ( $y$-map) and the nominal Planck position. 


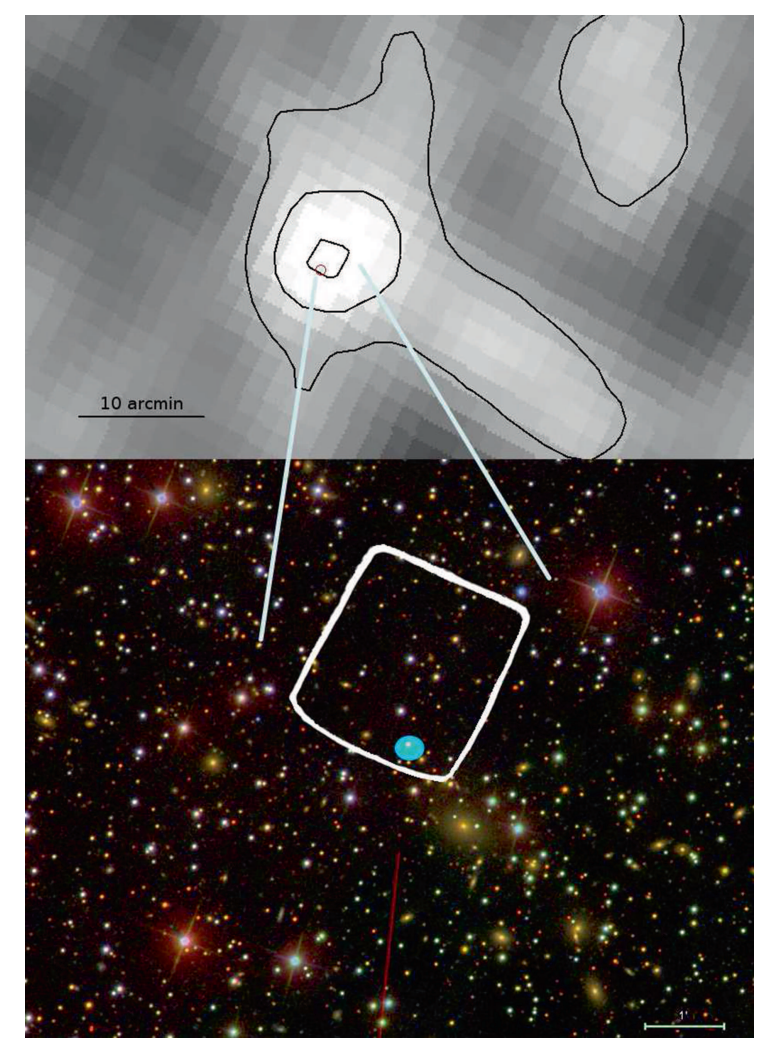

Fig. 7. Compton $y$-map (MILCA) and zoomed RGB SDSS image of the region around PSZ2 G069.35-15.58. The black contours correspond to the $3,6,9 \times 10^{-6}$ levels of the Compton $y$-map in this area where the smal red circle indicate the nominal Planck position from the PSZ2 catalogue. In the RGB image, the white contour corresponds to the $9 \times 10^{-6}$ level and the PSZ2 coordinates. The BCG of this system is clearly placed at less than $1^{\prime}$ to the south-west from the nominal Planck pointing. Potential cluster members are consistent with a $z_{\text {phot }} \sim 0.09$ and occupy the area of $\sim 12^{\prime} \times 12^{\prime}$.

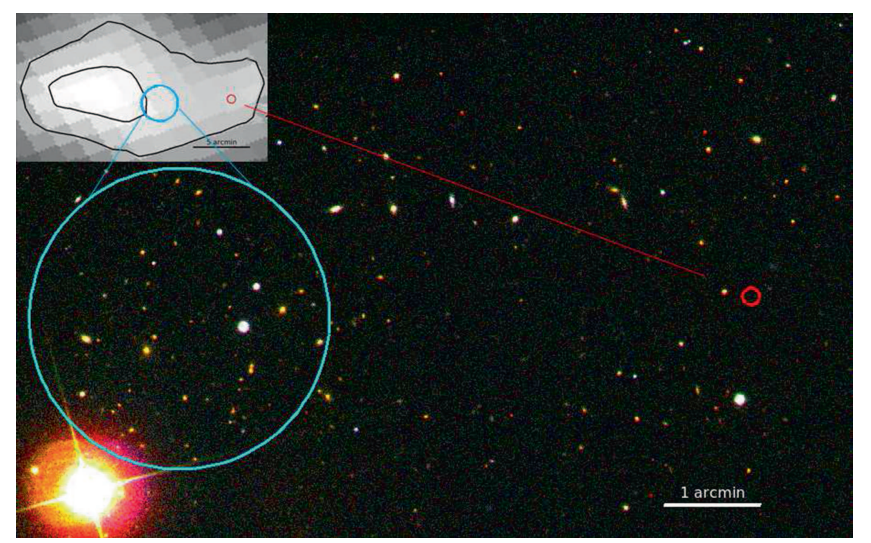

Fig. 8. Optical counterpart of PSZ2 G231.41+77.48 is located at $\sim 5.7$ from the nominal Planck position (the red small circle). The clear shift in the distribution of the SZ signal (seeing in the MILCA contours) supports this association.

PSZ2 G270.78+36.83 and PSZ2 G270.88+37.23 These two PSZ2 sources are separated by a distance of $\sim 25^{\prime}$ (see Fig. 6, right panel). The optical counterpart of PSZ2 G270.88+37.23 is a rich cluster $(\mathrm{R}=15)$ at $z_{\mathrm{phot}} \sim 0.23$ catalogued by Wen et al. (2012) as WHL J110504.6-185639. However, PSZ2 G270.78+36.83 seems to be a multiple detection, potentially associated with two clusters at different redshifts along the line of sight and almost

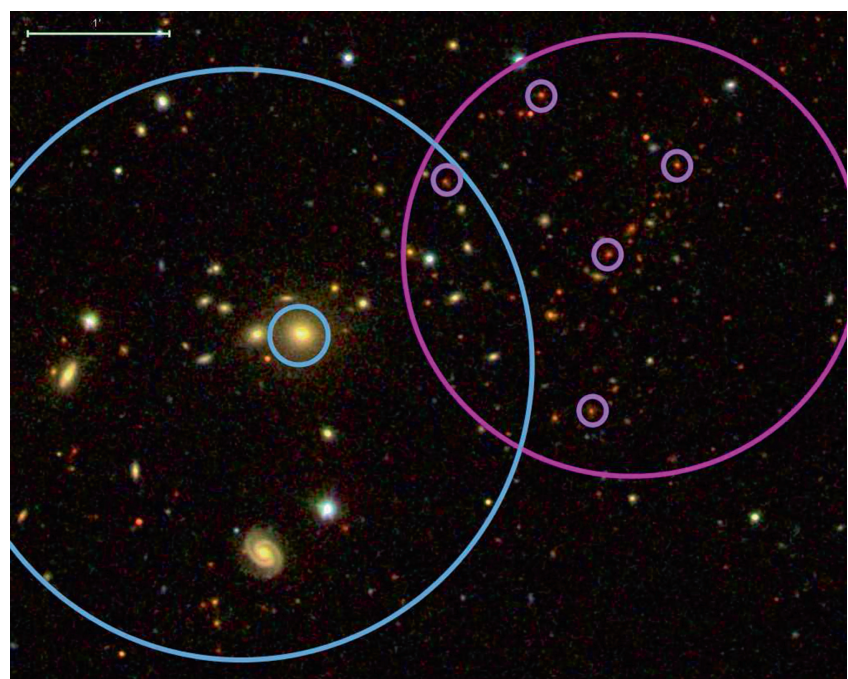

Fig. 9. RGB image of the SZ source PSZ1 G213.95+68.28. This target shows the presence of 2 possible optical counterparts at various redshifts. One at low redshift, $z_{\text {spec }}=0.10$ (blue circle), and a second cluster in the background at $z_{\text {spec }}=0.6$ (magenta circle). Spectroscopic cluster members are indicated with small circles. For both clusters we identify more than 20 spectroscopically confirmed members.

overlapping in the sky plane. One of these two clusters is a system at $z \sim 0.22$, while the second clump is a system at $z_{\text {phot }} \sim 0.52$. Both clusters probably contribute to the SZ emission, although their separate contribution requires further investigation. In total, we find three cases like this, where multiple counterparts are associated with a single SZ source. We denote these sources with a special symbol in the Table A.1.

\section{Results for the Planck PSZ1 catalogue}

We also inspected SDSS DR12 data in order to update the spectroscopic redshift information of PSZ1 clusters that were already confirmed photometricaly (Planck Collaboration XXXII 2015). We find 71 cluster fields in the SDSS footprint, but only 48 of these also have spectroscopic coverage. We have been able to retrieve spectroscopic information for 33 clusters, namely cases where likely members were spectroscopically observed. Almost half of the clusters have only one or two galaxy members with spectra. However, in five cases we find more than 10 members. In all 33 identifications, spectroscopic redshifts are in good agreement with the previous photometric data. No significant discrepancy is detected. The main reason for this is that an important fraction of these previous identifications belong to clusters retrieved through the cross-correlation of the original PSZ1 catalogue with the SDSS-III photometric cluster catalogue by Wen et al. (2012).

Additionally, we inspected a few still unconfirmed PSZ1 clusters inside the SDSS DR12 data and we have been able to identify the optical counterparts for the Planck SZ source PSZ1 G213.95+68.28. There are two possible identifications for this target, both of which are spectroscopically confirmed (see Fig. 9). This is a new case of multiple detection with two clear clusters at different redshifts along the line of sight. The low- $z$ cluster is at $z \sim 0.10$, showing about 50 likely members, 10 of which are spectroscopically confirmed. This low $-z$ cluster coincides with a compact cluster in the background, at $z \sim 0.601$. In fact, PSZ1 G213.95+68.28 is a rich structure with five cluster members at $0.595<z_{\mathrm{spec}}<0.603$ and 12 likely members showing a $z_{\text {phot }} \sim 0.6_{-0.1}^{+0.1}$. However, both systems are inside the peak of the 
Planck SZ detection in the $y$-maps and thus both are probably contributing to the SZ emission.

The identification of the BCG in clusters at low redshift is straightforward. However, in clusters at $z>0.5$ the BGC is not so clear. In general, clusters at high redshift are young and not so evolved structures and have less luminous and smaller BCGs than clusters at lower $z$ (Ascaso et al. 2011). In such cases, several galaxies are typically found with very similar magnitudes in the core of the clusters. Therefore, for these high redshift clusters we provide the coordinates of the average position of all spectroscopically confirmed members.

Our new spectroscopic redshifts for the PSZ1 sources are presented in Table A.3.

\section{Conclusions}

We carried out a detailed study of Planck PSZ2 targets using optical information from SDSS DR12. The technique presented in this work also combines the information from the Compton $y$-maps. We implement restrictions concerning the optical richness and distance from the nominal Planck pointing to validate the cluster counterparts. Together, this procedure allows for a more robust identification of optical counterparts compared to simply crossmatching with existing SDSS cluster catalogues that have been constructed from earlier SDSS Data Releases.

Using this method we confirmed 37 new Planck PSZ2 clusters. We also identified 17 more clusters in the SDSS images potentially associated with the corresponding SZ emission. However, this cluster sample does not completely follow the distance/richness requirements. These clusters could contribute somehow to the corresponding SZ emission but further investigations (mainly multi-object spectroscopy to obtain dynamical masses) are needed.

For 11 of the 37 new clusters we present spectroscopic redshifts (for 9 clusters from SDSS DR12 database, and 2 clusters from our long-slit observations using ACAM/WHT). Three SZ sources present multiple optical counterparts. For these cases, we find several clusters along the line of sight of the SZ signal.

Finally, we present updated spectroscopic information for 34 Planck PSZ1 sources, 33 of which have been previously confirmed photometrically. Additionally, we validate the cluster PSZ1 G213.95+68.28. This is a PSZ1 source showing two clusters along the line of sight at $z=0.101$ and $z=0.601$. The work presented is part of a more general programme to validate Planck SZ sources optically, trying to determine the purity and efficiency of cluster detections in Planck SZ maps.

Acknowledgements. We thank Monique Arnaud for helpful comments. This research has made use of the following databases: the SZ-Cluster Database operated by the Integrated Data and Operation Center (IDOC) at the IAS under contract with CNES and CNRS; and the SDSS. Funding for the Sloan Digital Sky Survey (SDSS) has been provided by the Alfred P. Sloan Foundation, the Participating Institutions, the National Aeronautics and Space Administration, the National Science Foundation, the U.S. Department of Energy, the Japanese Monbukagakusho, and the Max Planck Society. This research has been carried out with telescope time awarded by the CCI International Time Programme at the Canary Islands observatories (programme ITP13-8). The article is based on observations carried out at the William Herschel Telescope operated on the island of La Palma by the ISAAC Newton Group of Telescopes in the Spanish ORM of the IAC. This work has been partially funded by the Spanish Ministry of Economy and Competitiveness (MINECO) under the projects ESP2013-48362-C2-1-P and AYA201460438-P. AS and RB acknowledge financial support from the Spanish Ministry of Economy and Competitiveness (MINECO) under the 2011 Severo Ochoa Programme MINECO SEV-2011-0187. HL was supported by Estonian Research Council (ETAg) projects PUT1627, IUT26-2 and IUT40-2, and by the Centre of Excellence "Dark side of the Universe" (TK133) financed by the European Union through the European Regional Development Fund. RFJvdB acknowledges support from the European Research Council under FP7 grant number 340519 .

\section{References}

Abell, G. O. 1958, ApJS, 3, 211

Aihara, H., Allende Prieto, C., An, D., et al. 2011, ApJS, 193, 29

Ascaso, B., Aguerri, J. A. L., Varela, J., et al. 2011, ApJ, 726, 69

Barrena, R., Streblyanska, A., Ferragamo, A. et al. 2018, A\&A, 616, A42

Blanton, M., \& Roweis, S. 2007, AJ, 133, 734

Blanton, M., et al. 2003, ApJ, 592, 819

Borgani, S., \& Guzzo, L. 2001, Nature, 409, 39

Boschin, W., Barrena, R., Girardi, M., \& Spolaor, M. 2008, A\&A, 487, 33

Buddendiek, A., Schrabback, T., Greer, C. H., et al. 2015, MNRAS, 450, 4248

Eddington, A. S. 1913, MNRAS, 73, 359

Gladders, M. D., \& Yee, H. K. C. 2000, AJ, 120, 2148

Hao, J., McKay, T. A., Koester, B. P., et al. 2010, ApJS, 191, 254

Menanteau, F., \& Hugue, J. P. 2009, ApJ, 694, 136

Piffaretti, R., et al. 2011, A\&A, 534, A109

Planck Collaboration VIII. 2011, A\&A, 536, A8

Planck Collaboration XX. 2014, A\&A, 571, A20

Planck Collaboration XXI. 2014, A\&A, 571, A21

Planck Collaboration XXIX. 2014, A\&A, 571, A29

Planck Collaboration XXXII. 2015, A\&A, 581, A14

Planck Collaboration XXII. 2016, A\&A, 594, A22

Planck Collaboration XXVII. 2016, A\&A, 594, A27

Planck Collaboration Int. IV. 2013, A\&A, 550, A130

Planck Collaboration Int. VIII. 2013, A\&A, 550, A134

Planck Collaboration Int. XXVI. 2015, A\&A, 582, A29

Planck Collaboration Int. XXXVI. 2016, A\&A, 586, A139

Rozo, E., \& Rykoff, E. S. 2014, ApJ, 783, 80

Rozo, E., Rykoff, E. S., Evrard, A., et al. 2009a, ApJ, 699, 768

Rozo, E., Rykoff, E. S., Koester, B. P., et al. 2009b, ApJ, 703, 601

Rykoff, E. S., Rozo, E., Busha, M. T., et al. 2014, ApJ, 785, 104

Sunyaev, R. A., \& Zeldovich, Y. B. 1972, Comm. Astrophys. Space Phys., 4, 173

Tempel, E., Tamm, A., Gramann, M., et al. 2014, A\&A, 566, A1

van der Burg, R. F. J., Aussel, H., Pratt, G. W., et al. 2016, A\&A, 587, A23

Vikhlinin, A., Kravtsov, A. V., Burenin, R. A., et al. 2009, ApJ, 692, 1060

Wen, Z. L., Han, J. L., \& Liu, F. S. 2012, ApJS, 199,34 


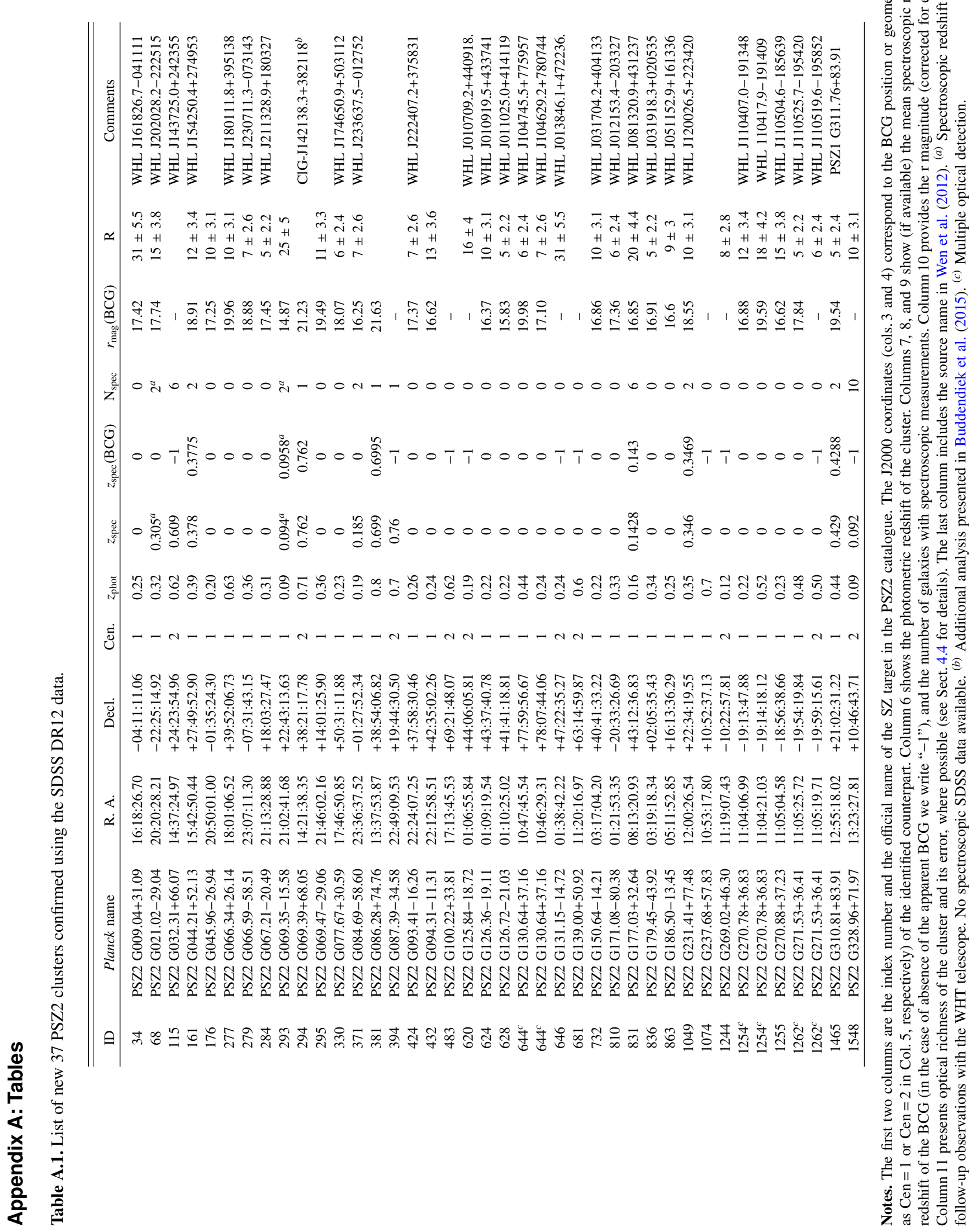


A. Streblyanska et al.: Validation of Planck SZ source cluster catalogues

Table A.2. List of 17 optical clusters potentially associated with the corresponding PSZ2 target.

\begin{tabular}{|c|c|c|c|c|c|c|c|c|c|c|}
\hline ID & Planck name & R. A. & Decl. & Cen. & $z_{\text {phot }}$ & $z_{\text {spec }}$ & $z_{\text {spec }} \mathrm{BCG}$ & $\mathrm{N}_{\text {spec }}$ & $r_{\mathrm{mag}}$ & Comments \\
\hline 126 & PSZ2 G036.36+16.01 & $17: 59: 42.69$ & $+10: 07: 43.70$ & 1 & 0.13 & $0.128^{a}$ & $0.1278^{a}$ & $3^{a}$ & 16.21 & \\
\hline 327 & PSZ2 G076.55+60.29 & $14: 52: 00.51$ & $+44: 31: 21.31$ & 2 & 0.29 & 0.287 & -1 & 4 & 0 & WHL J145206.4+443235 \\
\hline 421 & PSZ2 G092.69+59.92 & $14: 26: 08.53$ & $+51: 14: 14.15$ & 2 & 0.50 & 0.461 & -1 & 3 & 0 & \\
\hline 465 & PSZ2 G098.62+51.76 & 14:50:05.70 & $+59: 19: 51.91$ & 1 & 0.31 & 0.298 & 0.2985 & 1 & 18.16 & WHL J145005.7+591952 \\
\hline 546 & PSZ2 G112.69+33.37 & $16: 19: 49.38$ & $+79: 06: 23.53$ & 1 & 0.51 & 0 & 0 & 0 & 19.12 & WHL J161949.3+790624 \\
\hline 673 & PSZ2 G137.24+53.93 & $11: 40: 59.55$ & $+61: 07: 07.04$ & 1 & 0.48 & 0.473 & 0.4769 & 2 & 18.98 & WHL J114059.5+610707 \\
\hline 690 & PSZ2 G141.98+69.31 & $12: 12: 40.63$ & $+46: 21: 23.07$ & 1 & 0.70 & 0.714 & 0.7132 & 2 & 21.10 & WHL J121240.6+462123 \\
\hline 714 & PSZ2 G146.16-48.92 & 01:52:40.99 & $+11: 14: 30.90$ & 2 & 0.49 & 0.491 & -1 & 9 & 0 & \\
\hline 739 & PSZ2 G152.40+75.00 & $12: 13: 19.22$ & $+39: 46: 26.91$ & 1 & 0.42 & 0.453 & 0.4513 & 6 & 19.16 & WHL J121319.2+394627 \\
\hline 744 & PSZ2 G153.56+36.82 & $08: 44: 21.57$ & $+62: 17: 48.40$ & 2 & 0.12 & 0.122 & -1 & 10 & - & \\
\hline 812 & PSZ2 G171.48+16.17 & $06: 37: 43.56$ & $+43: 48: 59.12$ & 1 & 0.28 & 0 & 0 & 0 & 17.15 & WHL J063743.6+434859 \\
\hline 916 & PSZ2 G202.61-26.26 & $04: 59: 50.17$ & $-03: 16: 47.52$ & 1 & 0.23 & 0 & 0 & 0 & 17.12 & WHL J045950.2-031647 \\
\hline 917 & PSZ2 G202.66+66.98 & 11:07:30.89 & $+28: 51: 01.10$ & 1 & 0.48 & 0.483 & 0.4814 & 3 & 19.07 & WHL J110730.9+285101 \\
\hline 1510 & PSZ2 G320.94+83.69 & 13:00:05.73 & $+21: 01: 28.26$ & 1 & 0.45 & 0.462 & 0.4612 & 4 & 19.12 & WHL J130005.7+210128 \\
\hline
\end{tabular}

Notes. These targets do not fit the selection requirements of distance/richness to the Planck SZ detection. We refer to these sources as possible counterparts until further investigations are performed. Columns follow the same scheme as Table A.1. ${ }^{(a)}$ Spectroscopic redshift obtained from optical follow-up observations with the WHT telescope. No spectroscopic SDSS data available. 
Table A.3. List of confirmed clusters from PSZ1 with updated spectroscopic redshift.

\begin{tabular}{|c|c|c|c|c|c|}
\hline ID & Planck name & R. A. & Decl. & $z_{\text {spec }}$ & $\mathrm{N}_{\text {spec }}$ \\
\hline 52 & PSZ1 G020.82+38.03 & $16: 15.05 .44$ & $+07: 34: 55.38$ & 0.338 & 4 \\
\hline 97 & PSZ1 G035.55+34.15 & $16: 50: 35.73$ & $+16: 49: 31.27$ & 0.348 & 3 \\
\hline 142 & PSZ1 G046.98+66.62 & $14: 37: 40.29$ & $+30: 12: 00.27$ & 0.339 & 3 \\
\hline 143 & PSZ1 G047.44+37.39 & $16: 50: 20.40$ & $+26: 58: 21.42$ & 0.232 & 1 \\
\hline 257 & PSZ1 G078.39+46.13 & 16:09:01.48 & $+50: 05: 11.85$ & 0.400 & 1 \\
\hline 260 & PSZ1 G079.65+45.60 & $16: 10: 54.67$ & $+51: 13: 35.91$ & 0.392 & 2 \\
\hline 299 & PSZ1 G087.32+50.92 & $15: 26: 13.83$ & $+54: 09: 16.6$ & 0.473 & 4 \\
\hline 307 & PSZ1 G090.63+33.50 & $17: 27: 46.53$ & $+61: 30: 27.82$ & 0.312 & 1 \\
\hline $312^{a}$ & PSZ1 G091.81-26.97 & $22: 45: 27.74$ & $+28: 09: 00.78$ & 0.344 & 15 \\
\hline 321 & PSZ1 G093.15+43.20 & $16: 05: 15.53$ & $+61: 04: 42.54$ & 0.384 & 3 \\
\hline $357^{b}$ & PSZ1 G099.84+58.45 & $14: 14: 47.18$ & $+54: 47: 03.57$ & 0.620 & 2 \\
\hline 392 & PSZ1 G107.67-39.80 & $00: 01: 17.36$ & $+21: 31: 22.04$ & 0.411 & 18 \\
\hline 404 & PSZ1 G110.08-70.23 & $00: 33: 53.14$ & $-07: 52: 10.35$ & 0.302 & 3 \\
\hline 410 & PSZ1 G112.38-32.88 & $00: 10: 51.39$ & $+29: 09: 40.15$ & 0.332 & 19 \\
\hline 418 & PSZ1 G114.34-60.16 & $00: 34: 28.16$ & $+02: 25: 22.61$ & 0.382 & 3 \\
\hline 427 & PSZ1 G116.48-44.47 & $00: 32: 08.22$ & $+18: 06: 25.28$ & 0.374 & 11 \\
\hline 441 & PSZ1 G120.14-44.43 & $00: 42: 36.61$ & $+18: 25: 33.25$ & 0.281 & 3 \\
\hline 448 & PSZ1 G122.98-35.52 & $00: 51: 38.60$ & $+27: 19: 59.89$ & 0.358 & 6 \\
\hline 461 & PSZ1 G126.00-49.62 & $00: 59: 36.76$ & $+13: 10: 20.84$ & 0.501 & 8 \\
\hline 487 & PSZ1 G135.24+65.43 & $12: 19: 12.20$ & $+50: 54: 35.04$ & 0.552 & 7 \\
\hline 505 & PSZ1 G140.46+54.27 & $11: 30: 08.72$ & $+59: 46: 32.15$ & 0.169 & 6 \\
\hline 576 & PSZ1 G165.94+50.48 & 10:01:38.77 & $+50: 00: 53.59$ & 0.172 & 2 \\
\hline 579 & PSZ1 G166.61+42.12 & 09:09:38.69 & $+51: 36: 15.13$ & 0.215 & 3 \\
\hline 660 & PSZ1 G198.50+46.01 & $09: 30: 51.19$ & $+28: 47: 00.26$ & 0.296 & 7 \\
\hline $696^{c}$ & PSZ1 G212.51+63.18 & $10: 52: 52.73$ & $+24: 14: 00.14$ & 0.529 & 5 \\
\hline 699 & PSZ1 G212.80+46.65 & $09: 44: 42.87$ & $+19: 27: 59.64$ & 0.367 & 1 \\
\hline 701 & PSZ1 G213.27+78.35 & $11: 59: 31.29$ & $+26: 14: 38.73$ & 0.306 & 3 \\
\hline $705^{d}$ & PSZ1 G213.95+68.28 & $11: 14: 41.68$ & $+24: 34: 50.65$ & 0.101 & 10 \\
\hline $705^{d}$ & PSZ1 G213.95+68.28 & $11: 14: 32.15$ & $+24: 35: 24.06$ & 0.601 & 5 \\
\hline 719 & PSZ1 G217.35+58.14 & $10: 34: 39.66$ & $+20: 32: 04.52$ & 0.476 & 2 \\
\hline 743 & PSZ1 G223.80+58.50 & $10: 41: 09.64$ & $+17: 30: 35.08$ & 0.337 & 2 \\
\hline 760 & PSZ1 G226.65+28.43 & $08: 56: 20.59$ & $+01: 46: 49.38$ & 0.724 & 1 \\
\hline 795 & PSZ1 G236.86+66.33 & $11: 21: 55.15$ & $+15: 48: 05.16$ & 0.675 & 1 \\
\hline 1093 & PSZ1 G311.76+83.91 & $12: 55: 18.02$ & $+21: 02: 31.22$ & 0.429 & 2 \\
\hline 1159 & PSZ1 G332.30+72.17 & $13: 26: 43.58$ & $+11: 17: 04.22$ & 0.089 & 14 \\
\hline
\end{tabular}

Notes. The first two columns provide the index number and official name of the cluster in the PSZ1 catalogue. The J2000 coordinates correspond to the BCG or geometrical centre of the identified counterpart. Columns 5 and 6 show the new cluster redshifts and the number of galaxy members with spectroscopic measurements. ${ }^{(a)}$ Centre of the main group. This system consists of two groups at $z 0.344$ and 0.339 . ${ }^{(b)}$ Centre of the main rich cluster. The second nearby cluster has $z_{\text {spec }}$ of $0.579 .{ }^{(c)}$ Updated coordinates (centre of concentration). ${ }^{(d)}$ Double detection. 